\title{
Genetic variants in thyroid cancer distant metastases
}

\section{Dear Editor,}

Thyroid carcinoma is the most rapidly increasing solid tumor in the United States; although there has been a great emphasis on the analysis of primary tumors, predictors of both late-stage progression and response to therapy are more poorly defined due in part to the scarcity of progressive metastatic tissues. Therapeutic evidence of mixed responses in metastatic lesions and the limited available genomic data suggest that distant metastases in thyroid cancer are heterogeneous, driven by known oncogenes and other pathways that also might be therapeutic targets. We analyzed the genomes of a small number of rare surgically resected metastatic thyroid cancer lesions along with paired normal and primary tumor samples when available, in an effort to better characterize progressive distant metastases. The findings confirm the presence of mutations to known tumor drivers (BRAF and $R A S)$ in metastatic samples. The results also identified the co-occurrence in predicted functional variants to the DNA damage repair (DDR) genes ATM and ERCC4 in metastatic lesions that did not show alterations of the MAPK pathway.

We examined the exomes of 19 samples (including 5 paired normal tissues) from 11 follicular cellderived thyroid cancer patients with surgically resected distant metastases by custom exomeSeq (Supplementary Materials and Methods, see section on supplementary data given at the end of this article). Tumors with different histopathologies were included and were confirmed by an expert thyroid pathologist (PW). All patients were treated with TSH suppression, eight of them received I-131 therapy and none of them received chemotherapy or kinase inhibitors. Overall, 19,299 unique variants in the 682 genes and genomic regions were identified by an exomeSeq custom panel. We sought to identify rare, conserved, exonic variants that were likely to have functional effects. Thus, we focused on 1742 exonic variants in exons of sequenced genes. We excluded synonymous variants and variants with higher than 0.01 minor allelic frequency in the 1000 Genomes
Project. We then filtered to include variants located in regions that were conserved through vertebrate evolution. These criteria produced a list of 349 variants in 199 genes. Unsupervised clustering using complete linkage and Euclidian distance identified clusters based on patients, suggesting that most variants were primarily associated with individuals rather than histology or tissue location (data not shown). We finally applied a filter to identify variants predicted or known to have a damaging (SIFT) or deleterious (PolyPhen) effect on the protein. Figure 1 summarizes the filtering strategy.

In the primary tumors of patients with distant metastases, 33 variants in 31 genes were identified by comparing with normal samples. We focused on genes affected in more than one tumor sample and found three such genes: BCR, BRAF and MAP4 (Fig. 2). Two variants that affect $B R A F$ in five primary tumor samples were identified. A Hürthle cell carcinoma (HCC) bore a BRAF T241M variant, although its functional significance is uncertain. The remaining samples with BRAF had mutations resulting in BRAF V600E and included two papillary thyroid cancers (PTC) primary tumors and a metastatic lesion, and one anaplastic thyroid cancer (ATC) metastatic lesion. We also identified four samples from four patients with an identical insertion resulting in a frameshift (chr22:23653975 Indel: TCCGG) in BCR including three primary tumors and one metastasis. The primary tumor samples were from follicular (FTC) and HCC, and a metastatic lesion from BRAF V600E PTC. Finally, three primary tumors had an identical mutation (R1112P) in MAP4. Two of the tumors are from patients with HCC, and the third is a PTC with a concurrent BRAFV600E mutation. BCR and MAP4 are functionally involved in cytoskeletal dynamics, although those roles and functional implications of the variants identified are not completely characterized.

In the metastatic tissues, we identified thirty variants in twenty-eight genes after filtering unique vs normal tissues. Variants in three genes, ATM, BRAF and ERCC4,

Published by Bioscientifica Ltd 


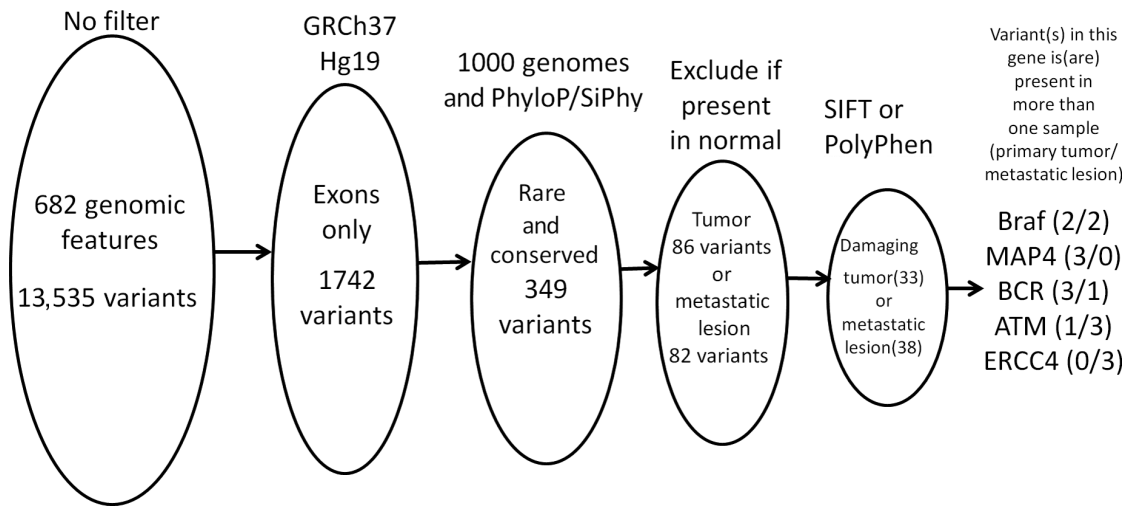

\section{Figure 1}

Schematic of the filtering criteria used on the genomic data to identify genes mutated in pathological samples. Five genes met an additional requirement that the gene had to be present in more than one sample. were present in more than one sample (Fig. 2). Two variants and a deletion in ATM were distributed among five samples from four patients. A variant that results in an R3008C transition (CHR11:108236086) was found in a primary tumor and metastatic lesion, but not in matched normal tissue from the same patient. Variant CHR11:108236153 resulting in a G3030E was found in three samples from three different patients. Both ATM variants occur in the C-terminal FATC domain of the protein. We also identified a deletion in ATM resulting in a frameshift. Additionally, we identified two variants in the ERCC4/XPF tumor suppressor gene. Variant CHR16:14041714 was found in a metastatic lesion from HCC and was concurrent with ATM variant CHR11:108236086. The second variant as identified in two metastatic samples from two patients and is concurrent with ATM variant CHR11:108236153.

We compared our results in primary tumors with those of the TCGA project on differentiated PTC (Cancer Genome Atlas Research Network 2014). Approximately, $60 \%$ of TCGA samples have a BRAF V600E mutation and $13.5 \%$ RAS mutation. In contrast, our study found $B R A F$ V600E in four samples and four samples with a RAS mutation among primary tumor and metastatic lesion samples. Although these differences may be due to the variety of histological types examined, a recent study focusing on poorly differentiated and anaplastic thyroid cancers (Landa et al. 2016) reported a similar rate of $B R A F$ V600E ( 33\%) and RAS ( 27\%).

Interestingly, five samples in this study had variants in the ATM tumor suppressor. In TCGA, only $1.3 \%$ of the differentiated PTC harbored variants in this gene, and none of those were located in the FATC domain. ATM mutations were reported by Landa and coworkers in $9 \%$ of samples, although none were located in the FATC domain. In that study, four ATM samples were concurrent with BRAF V600E, and one sample had concurrent $A T M$ and NRAS Q61R variants. Additional examination of publically available tissues (COSMIC) allowed us to identify 92 tumors and cell lines containing mutations of the ATM FATC domain. Five of those samples have

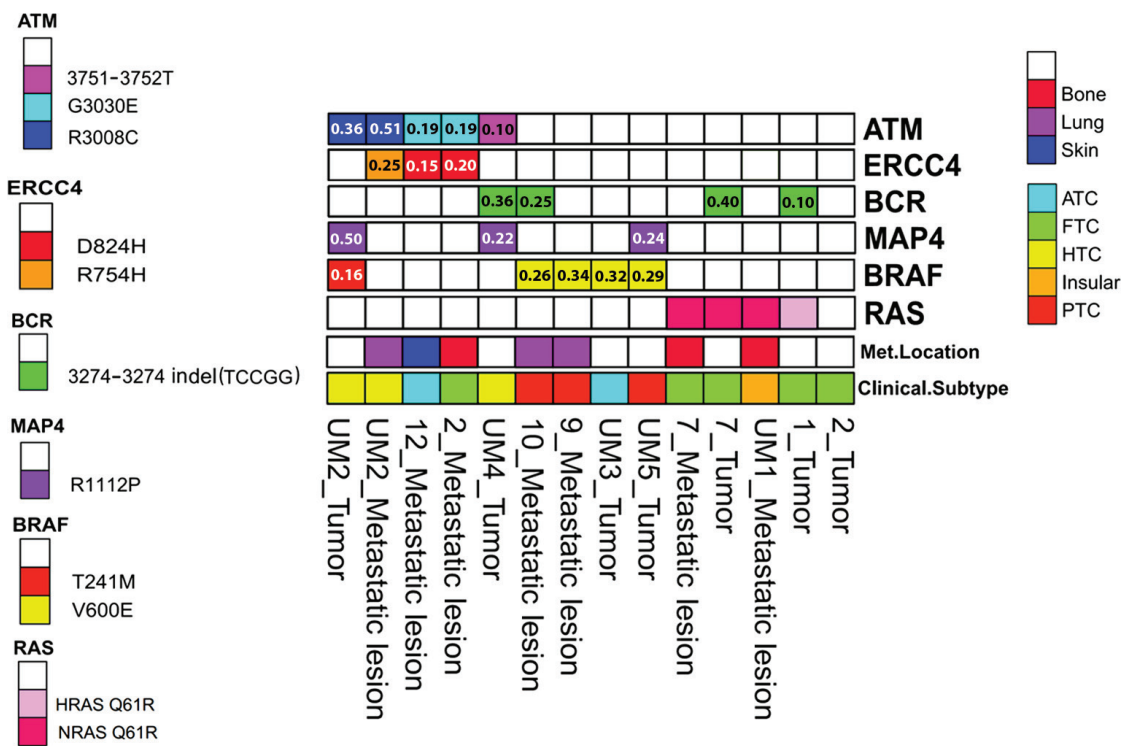

Figure 2

Candidate genes and variants found uniquely in more than one of 14 tumor tissue samples (primary or metastatic) vs normal tissues following filtering. One sample did not include a mutation in any of the analyzed genes. 
concurrent BRAF mutations, but only two were $B R A F$ $V 600 E$, one colon adenocarcinoma and one malignant melanoma. The FATC domain of ATM has been reported to be critical for telomere maintenance and effective DDR (Ogi et al. 2015). Additionally, the FATC domain has been implicated in NOTCH1 regulation and leukemia cell survival (Vermezovic et al. 2015). Codon 3008 appears to be a mutation hotspot, and this variant has been shown to have a dominant negative effect in vitro (Meyn 1999). The relationship between BRAF V600E and ATM FATC domain variants may suggest redundant functions that would require experimental verification.

We also identified variants in a second gene involved in the DDR, ERCC4/XPF, exclusively in metastatic samples and only co-occuring with ATM FATC variants. Altered expression of ERCC4 is common in colon cancer, where $\sim 55 \%$ of cases have epigenetic repression of the gene (Facista et al. 2012). Although it is not clear that ATM and ERCC4 cooperate in thyrocytes, such an interaction has been suggested in CLL cells in which co-occurrence of mutations in ERCC4 and ATM predicted synthetic lethality to ATR kinase inhibitors (Kwok et al. 2016).

The PTC TCGA study identified DDR variants in $~ 6.5 \%$ of samples, and no case had mutations to multiple DDR components (Cancer Genome Atlas Research Network 2014). In contrast, Landa and coworkers reported in poorly differentiated tumors that $38 \%$ of samples contain a mutation in at least one DDR gene, most commonly TP53, and in $\sim 6 \%$ of cases, there were mutations to multiple DDR genes (Landa et al. 2016). A recent study (Sohn et al. 2016) analyzed distant metastases and primary tumors from a group of well-differentiated PTC and FTCs using a targeted gene panel. That study found that $B R A F$ and $R A S$ mutations were largely conserved between primary tumors and distant metastases, but did not identify ATM variants or examine ERCC4/XPF as part of the panel. In this study, the ATM and ERCC4 variants occurred in FTC, HCC or ATC tumors, whereas none were noted in PTC samples. Finally, due to its relevance in thyroid cancer, we examined primary tumor samples from four (10, 12, UM2 and UM4) patients with adequate residual DNA for TERT promoter mutations by Sanger sequencing. One C228T mutation was identified (UM4), which did not have a variant in ERCC4 or a FATC domain variant in ATM. These data suggest that mutations in DDR-related genes may be enriched in poorly differentiated and non-PTC progressive metastatic thyroid cancers, although the small samples size of this study requires further work to test this hypothesis.

It is important to note that these findings are in a small number of samples from a diverse group of tumors and require confirmation to rule out falsepositive results. Moreover, the use of a broader highdensity sequencing platforms may lead to additional findings and functional studies are needed for variants of uncertain functional significance. We believe these descriptive data provide a scaffold for further studies to better define therapeutic targets for patients with progressive metastatic thyroid cancer.

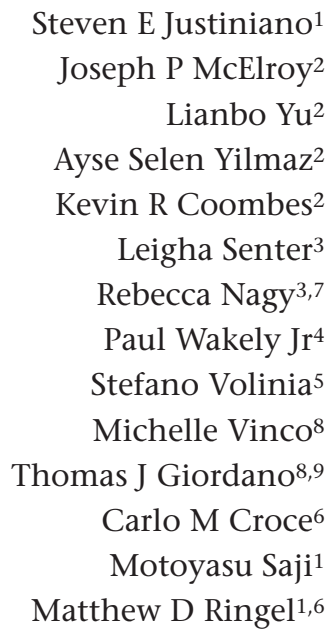

${ }^{1}$ Division of Endocrinology, Diabetes, and Metabolism, The Ohio State University, Columbus, OH, USA ${ }^{2}$ Center for Biostatistics and Department of Bioinformatics, The Ohio State University, Columbus, OH, USA ${ }^{3}$ Division of Human Genetics, The Ohio State University, Columbus, OH, USA ${ }^{4}$ Department of Pathology,

The Ohio State University, Columbus, OH, USA

5Department of Morphology, Surgery and Experimental Medicine, University of Ferrara, Italy ${ }^{6}$ Department of Molecular Virology, Immunology, and Genetics, The Ohio State University Wexner Medical Center and Arthur G. James Comprehensive Cancer Center, Columbus, Ohio, USA

${ }^{7}$ Guardant Health, Inc, Redwood City, California, USA ${ }^{8}$ Department of Pathology, University of Michigan, Ann Arbor, Michigan, USA

${ }^{9}$ Comprehensive Cancer Center, University of Michigan, Ann Arbor, Michigan, USA

(Correspondence should be addressed to M D Ringel; email: matthew.ringel@osumc.edu)

Supplementary data

This is linked to the online version of the paper at http://dx.doi.org/10.1530/ ERC-16-0351.

Published by Bioscientifica Ltd. 
Declaration of interest

The authors declare that there is no conflict of interest that could be perceived as prejudicing the impartiality of the research reported.

\section{Funding}

This research was supported by NIH grant to M D R (P01 CA124570 and P50CA168505) and an S grant to S V from AIRC (Associazione Italiana per la Ricerca sul Cancro).

\section{References}

Cancer Genome Atlas Research Network 2014 Integrated genomic characterization of papillary thyroid carcinoma. Cell 159 676-690. (doi:10.1016/j.cell.2014.09.050)

Facista A, Nguyen H, Lewis C, Prasad AR, Ramsey L, Zaitlin B, Nfonsam V, Krouse RS, Bernstein H, Payne CM, et al. 2012 Deficient expression of DNA repair enzymes in early progression to sporadic colon cancer. Genome Integrity 3 1-21. (doi:10.1186/2041-9414-3-3)

Kwok M, Davies N, Agathanggelou A, Smith E, Oldreive C, Petermann E, Stewart G, Brown J, Lau A, Pratt G, et al. 2016 ATR inhibition induces synthetic lethality and overcomes chemoresistance in TP53or ATM-defective chronic lymphocytic leukemia cells. Blood $\mathbf{1 2 7}$ 582-595. (doi:10.1182/blood-2015-05-644872)

Landa I, Ibrahimpasic T, Boucai L, Sinha R, Knauf JA, Shah RH, Dogan S, Ricarte-Filho JC, Krishnamoorthy GP, Xu B, et al. 2016 Genomic and transcriptomic hallmarks of poorly differentiated and anaplastic thyroid cancers. Journal of Clinical Investigation 126 1052-1066. (doi:10.1172/JCI85271)

Meyn MS 1999 Ataxia-telangiectasia, cancer and the pathobiology of the ATM gene. Clinical Genetics 55 289-304. (doi:10.1034/j.13990004.1999.550501.x)

Ogi H, Goto GH, Ghosh A, Zencir S, Henry E \& Sugimoto K 2015 Requirement of the FATC domain of protein kinase Tel1 for localization to DNA ends and target protein recognition. Molecular Biology of the Cell 26 3480-3488. (doi:10.1091/mbc.E15-05-0259)

Sohn SY, Park WY, Shin HT, Bae JS, Ki CS, Oh YL, Kim SW \& Chung JH 2016 Highly concordant key genetic alterations in primary tumors and matched distant metastases in differentiated thyroid cancer. Thyroid 26 672-682. (doi:10.1089/thy.2015.0527)

Vermezovic J, Adamowicz M, Santarpia L, Rustighi A, Forcato M, Lucano C, Massimiliano L, Costanzo V, Bicciato S, Del Sal G, et al. 2015 Notch is a direct negative regulator of the DNA-damage response. Nature Structural \& Molecular Biology 22 417-424. (doi:10.1038/nsmb.3013)

Received in final form 16 August 2016

Accepted 19 August 2016

Accepted Preprint published online 19 August 2016
() 2016 Society for Endocrinology Printed in Great Britain
Published by Bioscientifica Ltd. 